\title{
THE TEACHING AND USES OF CONJOINT ANALYSIS AND THE ANALYTIC HIERARCHY PROCESS
}

\author{
Lawrence Bodin and Robert Krapfel \\ Robert H. Smith School of Business \\ University of Maryland \\ College Park, MD 20742 \\ Email: Ibodin@arhsmith.umd.edu
}

\begin{abstract}
Conjoint Analysis is a standard technique for market research and Conjoint Analysis uses painwise comparisons in some of its analyses. In this paper, we examine how AHP can be used along with Conjoint Analysis for market research and how the output from Conjoint Analysis can be used as input to the AHP for more global decision-making. Also, in this paper, some of the basic ideas in Conjoint Analysis are described and an example is presented.
\end{abstract}

\section{Introduction}

Conjoint Analysis, a standard technique for market research, and the Analytic Hierarchy Process (AHP), a standard decision-making procedure, are taught as part of the first year MBA curriculum at the Robert H. Smith School of Business at the University of Maryland. Conjoint Analysis is taught in the first course in Marketing and the AHP is taught in the first course in Quantitative Decision-Making. Until the 1999 Spring semester, there was no coordination in the teaching of these two topics. Since both approaches use pairwise comparisons, some of the MBA students inquired about the similarities and differences between these two approaches. The authors of this paper decided to coordinate the teaching of these two approaches by creating a common example and teaching in material to their classes on the same day. Thus, the students were presented both approaches worked out on the same example on the same day. This teaching experiment was carried out in April, 1999.

In preparing the materials for these classes, some interesting questions arose as to how the output from a Conjoint Analysis can be used as input to the AHP and how the AHP can be used as a complement to Conjoint Analysis for market research analysis. These ideas are explored in the last section of this paper. To begin, however, we present a brief description of Conjoint Analysis and work through a very simple example that uses both Conjoint Analysis and the AHP. There is a rich literature on Conjoint Analysis (Dolan, 1990; Louviere, 1988; Rangaswamy and Lilien, 1997). Since this meeting is on AHP, we believe that there is no need to discuss the question, "What is AHP?"

\section{What is Conjoint Analysis?}

Conjoint Analysis helps market researchers understand how individuals value features (or "attributes") of products or services and how these attributes affect the acceptability of these products. Conjoint Analysis is used to determine how consumers trade off different "levels" of these attributes in preferring one product to another. Some of the questions that Conjoint Analysis can be used to answer are as follows:

Which new products will be successful?

Which features of a product or service drive the purchase decision?

Do specific market segments exist for a product?

What advertising appeals will be most successful with these segments?

Will changes in product design increase consumer preference and sales? 
What is the optimal price to charge consumers for a product or service?

(These questions are adapted from the web sites of Sawtooth Software and SurveySite, two companies that produce Conjoint Analysis software.)

\section{Conjoint Analysis For Predicting Market Share}

Two Conjoint Analysis procedures for predicting market share are called the Metric Scoring Task and the Pairwise Comparison Task. These tasks are now briefly described.

\section{A. Metric Scoring Task}

In the Metric Scoring Task, the respondent is asked to evaluate each computer by use of a rating scale. This rating scale is commonly 1-5 or 1-9. The respondent is first asked to evaluate the desirability of each level of each feature independently. These scores are used for baseline internal consistency checks. The respondent is then presented with each product and asked to determine the respondent's preference for the product. Each product is a bundle of varying combinations of levels of the attributes. The experimental design may be either 1) a full factorial crossing all levels of the attributes if interaction effects are suspected or 2) a fractional factorial set of products (Computers A, B, and C in the example below) if interaction effects are not anticipated. The conjoint algorithms then compute utility values for each level of each attribute. These utility values are unitless index numbers that reflect relative contribution of the various attributes to the overall attractiveness of the bundle or product.

\section{B. Pairwise Comparison Task}

The Pairwise Comparison Task is the non-metric form of Conjoint Analysis. The Pairwise Comparison Task is carried out in the same manner as the Metric Scoring Task with the exception that in the Pairwise Comparison Task, the respondent simply rank-orders the products (bundles) by conducting pairwise comparisons two products at a time. Thus, in the Pairwise Comparison Task, the respondent declares that $\mathrm{A}$ is better than $\mathrm{C}, \mathrm{C}$ is better than $\mathrm{B}$, etc. The computational algorithms in Conjoint Analysis first convert the pairwise comparisons into absolute ranks and then process the ranks into utility values as described above. The non-metric form of the Conjoint Analysis places less of a cognitive burden on the respondent, and is generally favored when larger numbers of attributes and or levels of attributes are examined. The non-metric form of the Conjoint Analysis imposes limitations with respect to analysis. The utility values generated by both the Metric Scoring Task and the Pairwise Comparison Task can be used as input to other analyses described later in this paper.

\section{Portable Computer Example (Dolan, 1990)}

The following example was originally presented in Dolan, 1990. Suppose that an organization is trying to determine the consumer preferences for three different portable computers. The attributes of the three computers are as follows:

\begin{tabular}{lllll} 
& Weight & $\begin{array}{c}\text { Battery } \\
\text { Life }\end{array}$ & $\begin{array}{l}\text { Quality of } \\
\text { Resolution }\end{array}$ & Price \\
\hline Computer A: & $2 \mathrm{lbs}$ & 1 hour & Below Average & $\$ 2,000$ \\
Computer B: & $5 \mathrm{lbs}$ & 4 hours & Average & $\$ 3,000$ \\
Computer C: & $>5 \mathrm{lbs}$ & $\mathbf{8}$ hours & Average & $\$ 1,000$
\end{tabular}

Although this example is simple, it can be used to illustrate many of the features of Conjoint Analysis and AHP and how these two approaches can be integrated together.

The Conjoint Analysis is carried out for many respondents using the Metric Scoring Task and Pairwise Comparisons Task described above. Based on this analysis, the following values for the attributes in this example are determined. 


\begin{tabular}{cclc} 
Weight & $\begin{array}{c}\text { Battery } \\
\text { Life }\end{array}$ & \multicolumn{1}{c}{$\begin{array}{c}\text { Quality of } \\
\text { Resolution }\end{array}$} & Price \\
\hline 2 1 lbs.: 1.2 & 1 hour: 0.0 & Below Ave.: 0.0 & $\$ 1,000: 1.0$ \\
\hline 2-5 lbs.: 0.9 & 2 hours: 0.2 & Average: 0.4 & $\$ 2,000: 0.5$ \\
$>5$ lbs.: 0.0 & 4 hours: 1.5 & Above Ave.: 0.5 & $\$ 3,000: 0.0$ \\
& 8 hours: 1.5 & &
\end{tabular}

The value of each computer is the sum of the values of the attributes of the computer. As an example, the value of computer $A$ is found as follows:

Value of Computer 1 = Value of a 2 Ibs computer + Value of a 1-hour battery life + Value of a below-average resolution monitor + Value of a $\$ 2,000$ price $=1.2+0+0+.5=1.7$

In a similar manner, the value of Computer $B$ is computed to be 1.9 and the value of computer $C$ is computed to be 3.0.

Assuming that all products are available, the share of preference rule (Dolan, 1990) then uses the above information to give an estimate of the probability that a consumer will choose a computer and, by extension, the market share of each product. The following probabilities, interpreted as market shares for the computers. are computed as follows:

$$
\begin{aligned}
& \mathrm{P}[\text { Choosing Computer } \mathrm{A}]=1.7 /(1.87+1.9+3.0)=.26 \\
& \mathrm{P}[\text { Choosing Computer } \mathrm{B}]=1.9 /(1.87+1.9+3.0)=.29 \\
& \mathrm{P}[\text { Choosing Computer } \mathrm{C}]=3.0 /(1.87+1.9+3.0)=.45
\end{aligned}
$$

The following summarizes the above discussion (Dolan, 1990):

"The scenario simulation capability (of Conjoint Analysis) is a powerful tool with respect to assessing a new product introduction strategy. By describing a prospective new product on the salient attributes, one can obtain not only a market share estimate, but also an indication of which competitive products will be hurt most. This is achieved by first simulating the scenario of only the current competitive products being available and then the environment of current competitive products plus the prospective new product."

\section{AHP on the Portable Computer Example}

The sideways view of a very simple AHP tree for this example is given in Figure 1. The four criteria are Weight, Battery Life, Quality of Resolution and Price. The three alternatives are Computer A, Computer B and Computer C.
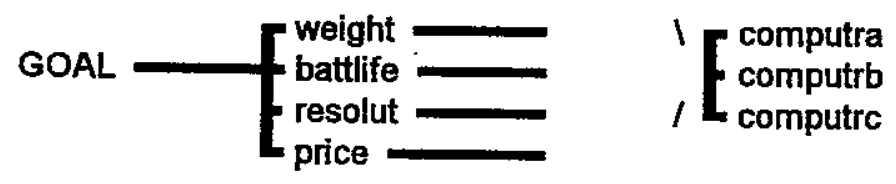

Figure 1: Sideway View of an AHP Tree for the Portable Computer Example 
We attempted to rank the three portable computers using the AHP where we used the utility values from the Conjoint Analysis as a basis to construct the pairwise comparisons. In the Conjoint Analysis, the value of each computer is the sum of the utility values of the attributes for the computer. Thus, it appears that the Conjoint Analysis weighs each attribute equally in determining the value of a computer. As such, the value of each pairwise comparison for the four criteria in the AHP is set equal to one. Then, we carried out pairwise comparisons of the three computers for each of the four criteria. We attempted to make the pairwise comparisons for these criteria to be representative of the values of the attributes determined in the Conjoint Analysis.

The results of this analysis are presented in Figure 2. In Figure 2, the numbers for the four criteria give the value that the AHP determined for each of the criteria for each of the computers. Thus, .183 is the value that the AHP determined for the criterion, Weight for Computer A. The Total AHP Weight for a computer is the sum of the four values attached to each of the four criteria for that computer. The Conjoint Analysis Share of Preference for each computer is the Value of a Computer that was determined in the Conjoint Analysis section of this paper.

\begin{tabular}{|c|c|c|c|c|c|c|}
\hline & Weight & $\begin{array}{l}\text { Battery } \\
\text { Life }\end{array}$ & $\begin{array}{l}\text { Quality of } \\
\text { Resolution }\end{array}$ & Price & $\begin{array}{c}\text { Total } \\
\text { AHP Weight }\end{array}$ & $\begin{array}{l}\text { Conjoint } \\
\text { Analysis } \\
\text { Share of Preference }\end{array}$ \\
\hline Computer A: & .183 & .015 & .023 & .056 & .277 & .26 \\
\hline Computer B: & .047 & .118 & .114 & .018 & .297 & .29 \\
\hline Computer $\mathrm{C}$ : & .020 & .118 & .114 & .177 & .429 & .45 \\
\hline
\end{tabular}

Figure 2: Results of the AHP Analysis for the Portable Computer Example

The results of this experiment are quite interesting. The Total AHP Weight and the Conjoint Analysis Share of Preference are reasonably close and, hence, the total AHP Weight gives a reasonable estimate of market share in the same way as the Conjoint Analysis Share of Preference gives a reasonable estimate of market share.

Some of the differences between the two approaches are now described. The AHP gives an actual ranking of the three alternatives for a person who is both the decision-maker and the respondent. Thus, if many decision-makers or respondents use the same AHP tree, their ranking of the alternatives may be different since their pairwise comparisons may be different. Each decision-maker or respondent can make a different decision on the ranking of the alternatives using AHP. Furthermore, the AHP gives an internal measure of consistency for the person (decision-maker and respondent) doing the analysis. If the person (decision-maker and respondent) is the consumer, then the ranking of the computers can give the consumer insight into the computer to purchase.

On the other hand, in a Conjoint Analysis, the respondent is not the decision-maker. In a Conjoint Analysis, the decision-maker uses a sample of hundreds (or thousands) of respondents is order to develop a single set of values for each of the attributes and each of the products. From these values, the estimated market share (or relative market acceptability) of each of the products is computed under the assumption that all of the products are introduced. Conjoint Analysis has its own way of determining consistency that can be considered a statistical test for outliers. In this case, Conjoint Analysis examines the answers of each respondent and determines if the answers of a respondent differ too much from the answers of the other respondents. In this case, the respondent's answers are rejected from the sample so that Conjoint Analysis gives an external measure of consistency.

\section{Possible Uses of Conjoint Analysis and the AHP}

In this section, we describe the possible use of AHP as a supplement to Conjoint Analysis in the determination of the share of preference of a set of products and the use of the results from Conjoint Analysis as input to an AHP. These ideas have not been tested and have to be regarded as proposals for possible discussion. 


\section{A. AHP in a Market Analysis}

As noted above, generally the purpose of conducting a Conjoint Analysis is different than the purpose of conducting an AHP. In a Conjoint Analysis, the respondents are not the decision-makers and simply provide data for the decision-maker. Conjoint Analysis can be regarded as a type of "statistical procedure" that merges together the responses of many respondents in order to determine the value of each of the products. Furthermore, in a Conjoint Analysis, the measure of consistency is across all of the respondents and outliers are eliminated from the sample.

On the other hand, a decision-maker is the only respondent in an AHP, The decision-maker gets his own individual ranking of the alternatives based on his individual preferences. The measure of inconsistency is internal to the decision-maker, based on the decision-maker's pairwise comparisons and independent of any other individual's pairwise comparisons. At this point, we are not discussing the team approach for conducting an AHP analysis.

The question then arises: Can the AHP be used to supplement a Conjoint Analysis in order to determine the share of preference of each product? We propose the following methodology. The decisionmaker (who is not a respondent) makes up the AHP tree. Each respondent then carries out the pairwise comparisons for the AHP. Then, in the same spirit as is currently used in Team Expert Choice, these responses are merged together to get average pairwise comparisons for the AHP tree. Then, the weights for the different alternatives are determined in the AHP. The weights for the alternatives become the estimated share of preference and overall measures of internal consistency can be computed

\section{B. Conjoint Analysis and the AHP for More Global Decision-Making}

In this situation, we are attempting to use the results from the Conjoint Analysis to serve as input to the AHP. In this case, the criteria used in the analysis are not simply the results from the Conjoint Analysis but the result of the Conjoint Analysis and other criteria that the company believe are important. The following illustrates this approach.

Suppose that the company has decided that the four major criteria for determining the products to introduce are the following:
i. Market Acceptability
ii. Profit Generated
iii. Ease of Procurement
iv. Risk

Market Acceptability gives an estimate of the share of preference of each alternative and is based on the results generated from the output of the Conjoint Analysis. Profit Generated is the profitability that each alternative can generate and is estimated through internal company analyses. Obviously, some of the results from the Conjoint Analysis can be used here as well. Ease of Procurement represents the difficulty in procuring the components that make up each alternative and assembling each alternative. Risk assesses how much risk is contained in assembling each alternative. Risk can consider such items as the possibility of attaining the estimated market share, difficulty in receiving the product from the place where the good is manufactured, etc. A possible simple AHP tree to analyze this problem is given in Figure 3.

The determination of the pairwise comparisons for the AHP for this example can involve the marketing, finance, manufacturing, accounting and legal departments of the company. The Team Expert Choice software system was specifically developed for this type of analysis. Conjoint Analysis can give critical marketing information to the decision-makers. The AHP can serve as a facilitator for discussion among the decision-makers from the various departments. This dialogue can be extremely useful to the decision-makers who is exploring the various alternatives and come up with an overall analysis. Furthermore, the output from the AHP can be used in an optimization model if more detailed analyses are 
needed. Together, the proper and innovative uses of Conjoint Analysis and the AHP can serve as a valuable decision-making tool.

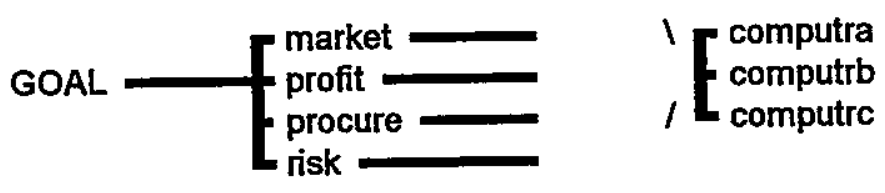

\section{Figure 3: AHP Tree for the Combined Conjoint Analysis - AHP Analysis for the Digital Camera Example}

As a note, the AHP tree presented in Figure 3 was presented to our MBA class with no pairwise comparisons filled in because we wanted to emphasis to the students that these pairwise comparisons may not be easy to determine and, thus, require care and consideration. In about 4 hours of class time, we were able to introduce both Conjoint Analysis and the AHP, construct the above examples, and show the students the interaction between these approaches. In general, the reaction to this material and its presentation was very favorable.

\section{References}

Dolan, R. J. (1990), Conjoint Analysis: A Manager's Guide, Case 9-590-059, Harvard Business School, Cambridge, MA. Reprints can be ordered from the Publication Division, Harvard Business School, Boston, MA 02163.

Louviere, J.J. (1988), “Analyzing Decision-Making: Metric Conjoint Analysis", Sage Publications, Newbury Park, CA.

Rangaswamy, A. and Lilien, G (1997), "Software Tools for New Product Development", Journal of Marketing Research, 34, February, 177-184. 